\title{
Comparison of clinical and social outcome of a) legally involuntarily admitted patients and $b$ ) of legally voluntarily admitted patients who feel coerced to admission across the EUNOMIA study sites Matthias Schützwohl*1 and Thomas Kallert ${ }^{1,2}$
}

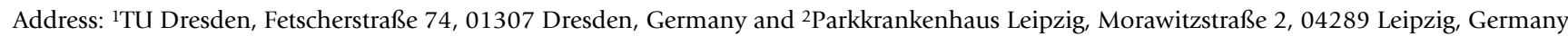

* Corresponding author

from WPA Thematic Conference. Coercive Treatment in Psychiatry: A Comprehensive Review

Dresden, Germany. 6-8 June 2007

Published: 19 December 2007

BMC Psychiatry 2007, 7(Suppl I):S98 doi:10.1 I86/I47I-244X-7-SI-S98

This abstract is available from: http://www.biomedcentral.com/I47I-244X/7/SI/S98

(c) 2007 Schützwohl and Kallert; licensee BioMed Central Ltd.

\section{Background}

To compare the clinical and social outcome between legally involuntarily admitted patients and legally voluntarily admitted patients who feel coerced at admission.

\section{Methods}

Within the EUNOMIA-study, $\mathrm{N}=2,586$ involuntarily admitted patients and $\mathrm{N}=830$ voluntarily admitted patients who felt coerced at admission were recruited across 13 sites in 12 European countries. Within the first ten days, 4 weeks, and 3 months after admission, patients were assessed on several variables including psychopathology (BPRS), level of functioning (GAF), QoL (MANSA), and satisfaction with treatment (CAT). The follow-up ratings of the two groups of patients will be presented and compared by means of multivariate analyses.

\section{Results}

Findings will inform of the medium-term outcome for the two groups of patients and of differences between the two groups.

\section{Conclusion}

Findings' implications will be discussed. 